\title{
An Innovative Design Of Packing Boxes: Quakeproof And Easy To Disassemble And Install
}

\author{
Tao Na \\ Wenzhou Vocational \& Technical College, Wenzhou. 325035 \\ taona@sohu.com
}

Keywords: Quakeproof. Demontierbarkeit.Packaging.Innovation.

\begin{abstract}
The new packing boxes which are quakeproof andeasy to disassemble and install include baseboard, the bottom of which have installation cabin. Besides, both sides of the interior of the cabin are furnished with the first slide and the second slide. the inside of the first slide is fitted with the first hinge shaft through the first slider. And The inside of the first hinged shaft is fitted with a third baffle, and the second slide is installed on the inside of the second slide. And the inner part of the second slider has a second hinge shaft, and the inner part of the second hinged shaft has the first baffle. Through the first slider and the second slider, the first baffle and the second baffle are installed in order to erect the baffle as a side wall when needed. When not in use, the baffle can be placed inside the installation bin by sliding rails. The third baffle can be installed between the first baffle and the second baffle can also facilitate the installation of the packing case.
\end{abstract}

\section{Research Status Of The Product}

Packing cases make it more convenient for people to transport and store products. Wooden cases and corrugated wood trays are generally used in packing cases, and tin drums or white iron drums are also used. Due to the convenience of packaging and relatively good storage, they are widely used in various fields.

The general structure of the current packing box is relatively simple, and there is no good assembly and disassembly structure, which is inconvenient for the assembly and disassembly of the box body. What's more, The current packing box function is relatively simple. They are mainly to storage goods and are not easy to transport. The internal shock absorption measures are relatively simple, and there is no good protection function for the goods. They can't transport and package fragile goods, which has limited the use of packing cases.

\section{Designing Scheme}

TourismThe purpose is to provide a new kind of packing box ,which can be quakeproof and easy to disassemble and install, to solve the related problems raised in the above background technology.

The inside of the first sliding rail of the design is fitted with the first hinge shaft through the first slider, and the inner part of the first hinged shaft has a third baffle. The second slider is installed on the inner side of the second slide, and the inner part of the second slider has a second hinge shaft. The inner part of the second hinged shaft of the design has the first baffle. The first baffle and the bottom end of the third baffle are fitted with an air pump, and both sides of the first baffle and the third baffle are set with a sliding groove. The inside of the first baffle and the third baffle is provided with the first protective cushion, and the inside of the first protective pad is equipped with the shock absorber. The output end of the design air pump is connected with the corresponding shock-absorbing air bag respectively through the airway. The second side plate is articulated at both ends of the top of the first baffle and the second side is hinged to the side of the first baffle.

Hanging rings are installed on the top and bottom of the first panel of the design. The design of the first baffle of the first baffle is hinged at the bottom of the third transition plate, and the third transition plate is connected to the side of the first baffle with a second hinge plate. The second 
hinge plate of the design is removed from the side of the third transition plate with the first hinge plate. One side of the first hinged plate of the design has a network wall, and the two sides of the wall are connected with the hanging ring. The bottom of the design installation warehouse has the storage bin. A battery is installed in the middle position of the bottom of the design bin. All the corners at the bottom of this design are installed with mounting blocks. L support plates are installed on both sides of the bottom of the design installation block.

A shock absorber is installed at the end of the design l-type support plate, which is far away from the mounting block, and the top of the shock absorber is fixed at the bottom of the storage bin. The design L support plate is removed from the bottom of the mounting block with a universal wheel. The top of the design floor is evenly fitted with a damping spring, and the top of the damping spring is fitted with a placement plate.

The top of the design placement plate has a slot. The second baffle is installed on both ends of the design floor, and the inner side of the second baffle is provided with a second protective pad. The two sides of the second baffle of the design have a sliding rod with the sliding groove, and two sets of slots are arranged on the top of the second baffle. The second baffle of the design is equipped with a cover plate, and both ends of the cover are hinged with l-shaped connecting rod. The design L type connecting rod is far away from the end of the cover plate and the plug is connected with the slot. The battery of is connected with the air pump through the wire.

\section{Assembly And Installation}

Regional This is a new kind of packing box , which can be quakeproof and easy to disassemble and install. The side of the receiving warehouse is installed with a warehouse door. And a vibration damper plate is arranged between the base plate and the damping spring. As is shown in Figure 1.

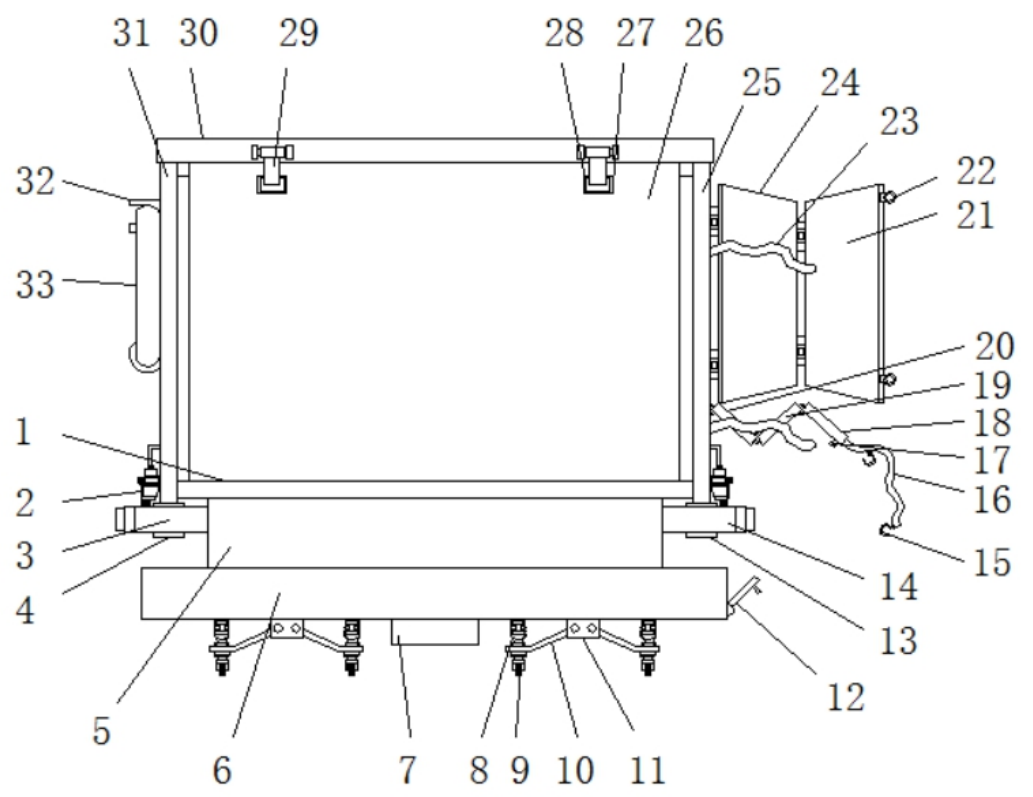

Figure 1. Packing Box and Storehouse Door

The inner sponge of the slot. The section of the slide rod and the chute is in trapezoid. The rotation Angle of the first hinged shaft and the second hinge axis is $0^{\circ}$ to $90^{\circ}$. As is shown in Figure 2. 


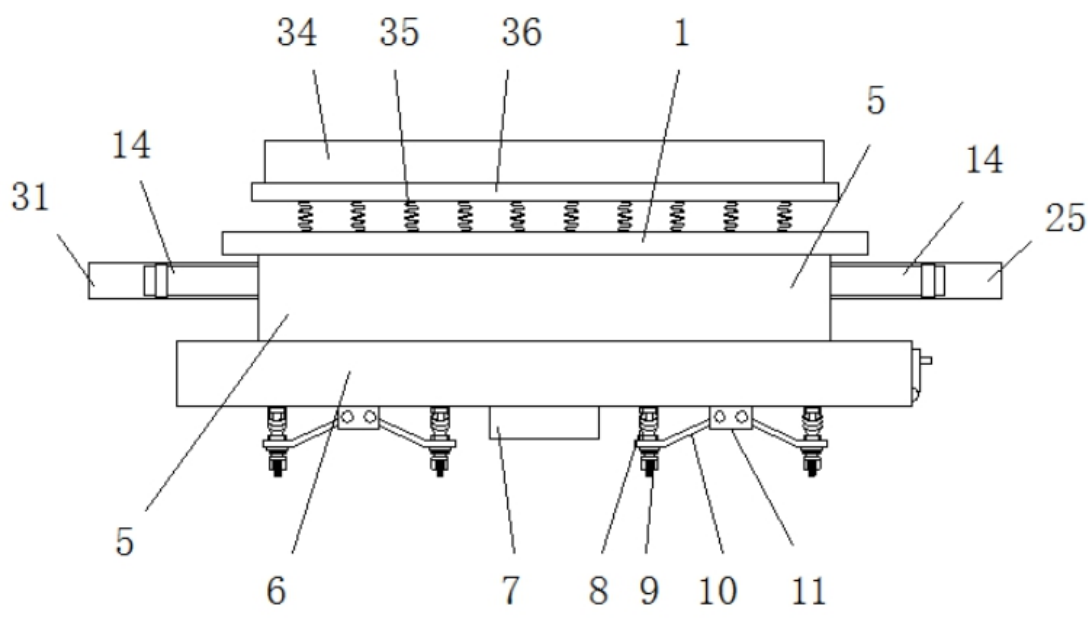

Figure 2 Slide and chute.

Two sets of fixed ropes are installed on both ends of the first baffle. The lateral top of the third baffle is hinged on both sides, and the middle position of the third baffle is hinged with a u-shaped push rod. There are two sets of limit holes in the one end of the design $U$ type push rod near the third baffle. The middle position of the top of the third baffle of the design is set with two sets of clasps. As is shown in Figure 3.

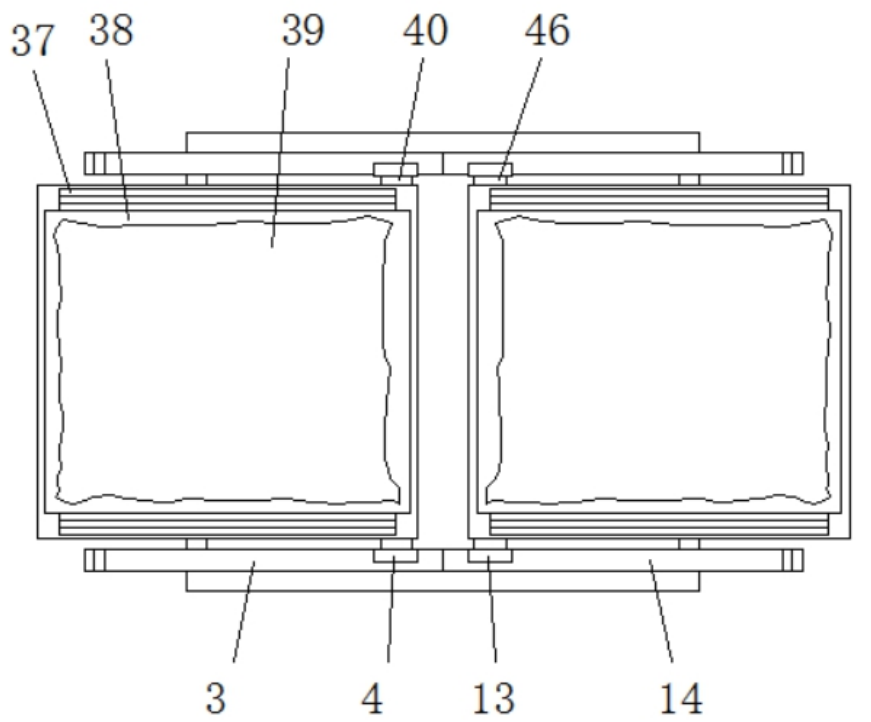

Figure 3 Two Sets of Fixed Ropes

Fixed bolts are installed on both ends of the second hinged plate far from the first hinged plate. And the first side of the plate is set with a fixed bolt that fits with the fixing bolt. As is shown in Figure 4.

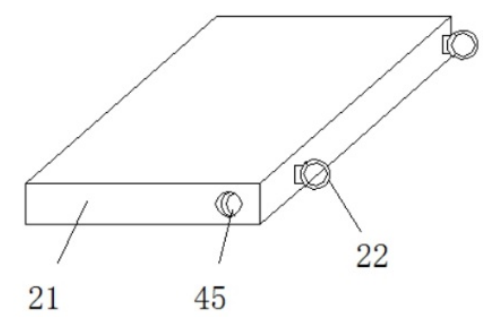

Figure 4 Fixed Bolt

Compared with the existing technology, the beneficial effect of this design is as follows: the new packing box is hinged by the first sliding block and the second slide rail with the first baffle and the second baffle. When used, the baffle can be erected as a side wall. As is shown in Figure 5. 


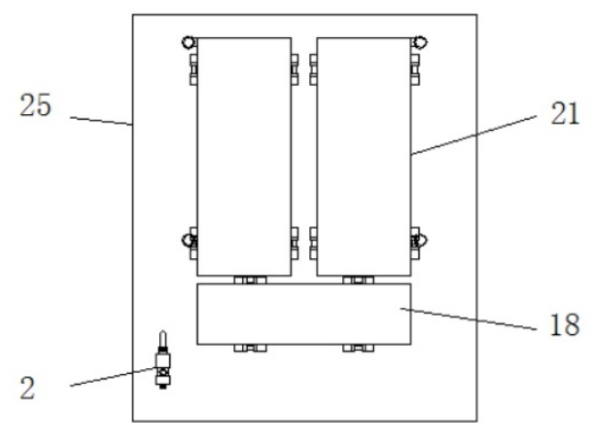

Figure 5 side wall

When not in use, the baffle can be placed inside the installation bin by sliding rails. And the third baffle can be installed between the first baffle and the second baffle to facilitate the installation of the packing box.

The packing case adopts the universal wheel structure, which makes the moving of the packing box more convenient, facilitates the movement of the packing box and plays the role of auxiliary transportation. As is shown in Figure 6.

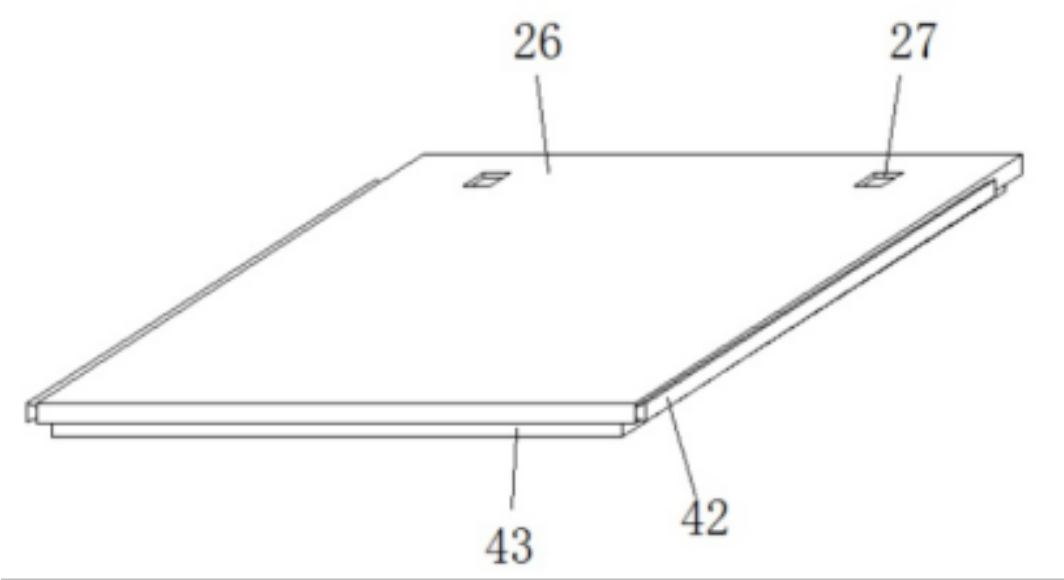

Figure 6 Device Removable

\section{Conclusion}

When packaging is not needed, the device can be used as a cart, thus effectively improving the functionality of the device. The damping structure is arranged on the universal wheel, which can reduce the vibration of the box body, and the damping spring at the top of the bottom plate can further improve the damping effect of the device. What's more, Air bags are installed on both the first and third baffles of the new packing box. When the fragile goods are needed to be packaged, air bags can be pumped through the air pump, so that the air bags on both sides will be clamped. That is to say, It can play the role of immobilization, and can play a better buffer and shock absorber. When the transportation space is insufficient, a temporary storage space can be formed through the side board, the transition board and the wall net of the first baffle. Thus, the use space of packing box is further improved, making the device more convenient to use.

\section{Acknowledgements}

2018 Zhejiang Province philosophy and social science planning (18NDJC133YB)

\section{References}

[1]C. Lijie and W. Jicheng, The Engineering Properties of the Product Colors. Journal of Packaging 
Engineering, vol.5. pp.5-6, 2012.

[2]Y.Suyun. Photography and Art. , Huaxia Press, Beijing, 2013.

[3]Z. Haidong and X. Lixin, Tips for Using Digital Cameras Well - Common Problems and Solutions for Digital Photography. Digital Printing, vol.7. pp .10-11. 2013.

[4]E. Heller and H. Eva, Color Culture, Central Compilation Press. Beijing 2011.

[5]K. Bing. Successful Print Advertising Design and Graphic Originality. Journal of Nanjing Art Institute (fine art and design edition), vol.01.pp.3-5. 2014.

[6]Z. Wenbin. Color Settings in Photoshop . Printing Technology r, vol. 25, pp. 36-38, 2014. 